\title{
ON THE TRIVIAL EXTENSION OF EQUIVALENCE RELATIONS ON ANALYTIC SPACES
}

\author{
BY \\ KUNIO TAKIJIMA AND TETSUTARO SUZUKI
}

\begin{abstract}
In this paper, we shall consider the problem: let $X$ be a (reduced) analytic space and $A$ a nowhere dense analytic set in $X$. And let $R$ be a proper equivalence relation on $A$ such that the quotient space $A / R$ is an analytic space, and $\tilde{R}$ the trivial extension of $R$ to $X$. Then, is $X / \tilde{R}$ an analytic space? To this, we have three sufficient conditions. Moreover, using this result we shall extend Satz 1 of H. Kerner [8].
\end{abstract}

1. Introduction. Let $\left(X,{ }_{X} 0\right)$ be an analytic space and $R$ an equivalence relation on $X$. Then the local ringed quotient space $\left(X / R,{ }_{X} O / R\right)$ is defined and the problem, whether $\left(X / R,{ }_{X} O / R\right)$ is an analytic space, is studied by H. Cartan, H. Holmann, B. Kaup and others.

In this paper, we shall consider the problem: let $X$ be a (reduced) analytic space and $A$ a nowhere dense analytic set in $X$. And let $R$ be a proper equivalence relation on $A$ such that the quotient space $A / R$ is an analytic space, and $\widetilde{R}$ the trivial extension of $R$ to $X$. Then, is $X / \widetilde{R}$ an analytic space? To this, we have

THEOREM. $X / \widetilde{R}$ is an analytic space, if one of the following three statements is satisfied:

(1) $R$ is finite.

(2) $A$ is contractible in $X$ and the canonical mapping $j: A / R \rightarrow X / \widetilde{R}$ is quasi-finite.

(3) $A$ is contractible and retractable in $X$.

Next, using Theorem, (3), we shall extend Satz 1 of H. Kerner [8] : let $X_{k}$ be a connected complex manifold, $A_{k}$ a contractible and retractable analytic set in $X_{k}$ and $R_{k}$ a proper equivalence relation on $A_{k}$ such that $A_{k} / R_{k}$ is an analytic space and $\operatorname{dim}_{a} R_{k}(a)>0$ for any $a \in A_{k}(k=1,2)$. Then, we have the following diagrams of analytic spaces:

Received by the editors July 16, 1974 and, in revised form, March 26, 1975.

AMS (MOS) subject classifications (1970). Primary 32D15.

Key words and phrases. Analytic space, proper equivalence relation, trivial extension of equivalence relation. 


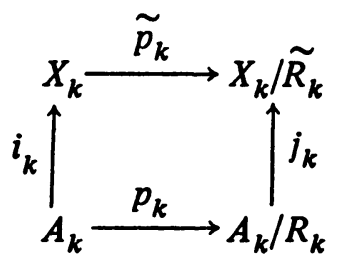

Here $p_{k}: A_{k} \rightarrow A_{k} / R_{k}, \tilde{p}_{k}: X_{k} \rightarrow X_{k} / \tilde{R}_{k}$ are natural projections, $i_{k}: A_{k} \rightarrow$ $X_{k}$ is the injection and $j_{k}: A_{k} / R_{k} \rightarrow X_{k} / \widetilde{R}_{k}$ is the canonical mapping. Let $r_{k}$ : $X_{k} \rightarrow A_{k}$ be the holomorphic retraction. Then, we have

THEOREM. Suppose that f.m.d. $r_{2} \geqslant \operatorname{dim} A_{1}+2$. If $X_{1} / \widetilde{R}_{1}$ and $X_{2} / \widetilde{R}_{2}$ are analytically equivalent, then the above two diagrams are analytically equivalent.

H. Kerner has treated the case that $r_{k}: X_{k} \rightarrow A_{k}$ is a weakly negative vector bundle and $R_{k}(a)=A_{k}$ for any $a \in A_{k}$.

2. Trivial extension of equivalence relations. Let $L$ be the category of local ringed spaces [6]: objects in $L$ are local ringed spaces and morphisms in $L$ are morphisms of local ringed spaces.

Definition 1. A commutative diagram of morphisms in $L$ :

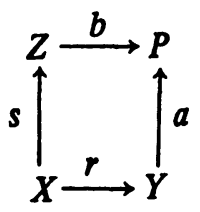

is called a pushout (and $P$ is called the pushout for $r$ and $s$ ), if for any object $A$ and morphisms $u: Y \rightarrow A, v: Z \rightarrow A$ in $L$ with $v \circ s=u \circ r$, there exists the unique morphism $p: P \rightarrow A$ such that $p \circ b=v$ and $p \circ a=u$.

Let $\left(X, X_{X} O\right)$ be a (reduced) analytic space and $R$ an equivalence relation on $X$. Then there exists the local ringed quotient space $\left(X / R,{ }_{X} \mathrm{O} / R\right)$ and the natural projection $p: X \rightarrow X / R$ is a morphism of local ringed spaces, where $X / R$ is the quotient topological space of $X$ by $R$ and ${ }_{X} O / R$, the structure sheaf on $X / R$, is defined as follows: for any open set $U \subset X / R,\left({ }_{X} O / R\right)(U):=\{f: U \rightarrow \mathrm{C}$, $\left.f \circ p \in \Gamma\left(p^{-1}(U),{ }_{X} 0\right)\right\}$.

DEFINITION 2. An equivalence relation $R$ on $X$ is called proper if for any compact set $K \subset X$, the $R$-saturated set $R(K)$ (i.e. the union of all equivalence classes meeting $K$ ) is also compact.

This condition is equivalent that $X / R$ is locally compact and the natural projection $p: X \rightarrow X / R$ is proper.

Definition 3. Let $A$ be a subset of $X$ and $R$ an equivalence relation on $A$. The trivial extension $\widetilde{R}$ of $R$ to $X$, an equivalence relation on $X$, is defined by 


$$
\tilde{R}(x):= \begin{cases}R(x), & \text { for } x \in A, \\ \{x\}, & \text { for } x \notin A,\end{cases}
$$

where $R(x), x \in A$, denotes the equivalence class by $R$ containing $x$.

Let $\left(A,{ }_{A} O\right)$ be a nowhere dense analytic set of $\left(X,{ }_{X} 0\right)$ and $R$ an equivalence relation on $A$. Then we have the local ringed quotient spaces $(A / R$, $\left.{ }_{A} O / R\right),\left(X / \widetilde{R},{ }_{X} O / \widetilde{R}\right)$. Let $p: A \rightarrow A / R, \tilde{p}: X \rightarrow X / \widetilde{R}$ be natural projections and $i: A \rightarrow X$ the injection. Then there exists the canonical mapping $j: A / R \rightarrow$ $X / \tilde{R}(\tilde{p} \circ i=j \circ p)$ and $j$ is a morphism in $L$.

LEMMA 1. $X / \widetilde{R}$ is the pushout for $i$ and $p$ in $L$.

Proof. For any object $Z$ and morphisms $u: A / R \rightarrow Z, v: X \rightarrow Z$ in $L$ with $v \circ i=u \circ p$, we define the mapping as follows: for any $\tilde{x} \in X / \widetilde{R}$, we put $\varphi(\tilde{x}):=v(x)\left(x \in \tilde{p}^{-1}(\tilde{x})\right)$. Then this is well defined. In fact $\tilde{p}(x)=\tilde{p}\left(x^{\prime}\right)$ $\left(x, x^{\prime} \in X\right)$ implies $v(x)=v\left(x^{\prime}\right)$. Now $\varphi$ is continuous with $v=\varphi \circ \tilde{p}$, and $u=$ $\varphi \circ j$ since $u \circ p=\varphi \circ j \circ p$ and $p$ is surjective.

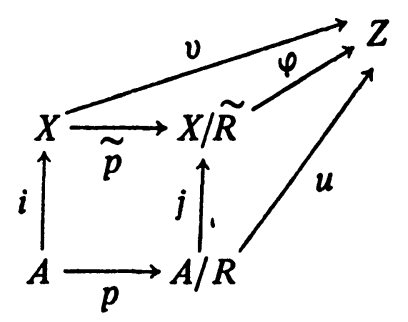

For any $f \in{ }_{Z} O_{\varphi(\tilde{x})}(\tilde{x} \in X / \tilde{R})$, there exists $\tilde{f} \in\left({ }_{X} O / \tilde{R}\right)_{\tilde{x}}$ with $v_{x}^{*}(f)=$ $\tilde{f} \circ \tilde{p}$. And we put $\varphi_{\tilde{x}}^{*}(f):=\tilde{f}$. Then $\varphi^{*}$ holds commutativity and is unique. Hence $X / \tilde{R}$ is the pushout in $L$ for $i$ and $p$. Q.E.D.

Definition 4. An analytic set $A \subset X$ is called contractible in $X$ if $A$ is nowhere discrete, compact and if there exist an analytic space $Y$ and a surjective proper holomorphic mapping $\psi: X \rightarrow Y$ such that $\psi(A)=: y_{A} \in Y$ and the restriction $\psi \mid(X-A) \rightarrow\left(Y-\left\{y_{A}\right\}\right)$ is biholomorphic.

Definition 5. An analytic set $A \subset X$ is called retractable if there exists a holomorphic retraction of $X$ to $A$ (i.e. a surjective hnlomorphic mapping $r$ : $X \rightarrow A$ with $r \mid A=\mathrm{id}_{A}$ ).

Definition 6. A morphism $f:\left(X,{ }_{X}\right) \rightarrow\left(Y,{ }_{Y}\right)$ in $L$ is called quasifinite if for any $x \in X,{ }_{x} O_{x} /\left(f_{x}^{*}\left(M_{f(x)}\right)\right)$ is a finite dimensional vector space over C, where $M_{f(x)}$ is the maximal ideal of ${ }_{Y} O_{f(x)}$.

Let $\left(A,{ }_{A} O\right)$ be an analytic set in $\left(X,{ }_{X} O\right)$ and $R$ a proper equivalence relation on $A$ such that $A / R$ is an analytic space. Using the results by B. Kaup [6] and the method of $\mathrm{H}$. Kerner [8], we shall show the sufficient conditions under which $X / \widetilde{R}$ is an analytic space. 
THEOREM 1. $X / \widetilde{R}$ is an analytic space, if one of the following statements is satisfied:

(1) $R$ is finite (i.e. every equivalence class of $A$ by $R$ is a finite set).

(2) $A$ is contractible in $X$ and the canonical mapping $j: A / R \rightarrow X / \widetilde{R}$ is quasi-finite.

(3) $A$ is contractible and retractable in $X$.

Proof. (1) From Lemma $1, X / \widetilde{R}$ is the pushout for the injection $i: A \rightarrow$ $X$ and the natural projection $p: A \rightarrow A / R$. Hence, by B. Kaup [6, Satz 1.8], $X / \widetilde{R}$ is an analytic space.

(2) If $A$ is contractible in $X, A$ is exceptional in $X$ in the sense of B. Kaup [6]. Hence, by Lemma 1 and B. Kaup [6, Aussage 1.11], $X / \widetilde{R}$ is an analytic space.

(3) $\widetilde{R}$ is proper since, for any compact set $K \subset X, \tilde{R}(K)=K \cup R(K)$ is also compact in $X$.

By the assumption, there exist an analytic space $Y$, a surjective proper holomorphic mapping $\psi: X \rightarrow Y$ and a holomorphic retraction $r: X \rightarrow A$. Then we have a surjective morphism $\tilde{r}: X / \widetilde{R} \rightarrow A / R$ with $\tilde{r} \circ \tilde{p}=p \circ r$. In fact, for any $\tilde{x} \in X / \widetilde{R}$, we put

$$
\tilde{r}(\tilde{x}):=p \circ r(x) \quad\left(x \in \tilde{p}^{-1}(\tilde{x})\right) .
$$

Then $\tilde{r}: X / \tilde{R} \rightarrow A / R$ is well defined.

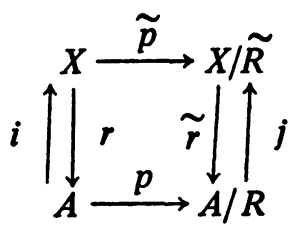

Now, we claim that $\left(X / \widetilde{R},{ }_{X} O / \widetilde{R}\right)$ is locally morph-separable (i.e. for any $\tilde{x} \in X / \widetilde{R}$, there exists an open neighborhood $U \subset X / \widetilde{R}$ such that $\Gamma\left(U,{ }_{X} O / \widetilde{R}\right)$ separates points of $U)$. Then $\left(X / \widetilde{R},{ }_{X} O / \widetilde{R}\right)$ is an analytic space by H. Cartan [1, Main Theorem].

Let $\tilde{x}$ be a point of $X / \tilde{R}$. We may assume that $\tilde{x} \in j(A / R)$. Then there exists an open neighborhood $V$ of $x:=\tilde{r}(\tilde{x})$ such that $\Gamma\left(V,{ }_{A} O / R\right)$ separates points of $V$ and also there exists an open neighborhood $O \subset Y$ of $y_{A}$ such that $\Gamma(O, Y)$ separates points of $O$. Since $W:=\psi^{-1}(O) \subset X$ is an open neighborhood of $A$, we have $\tilde{p}^{-1}(\tilde{p}(W))=W$, hence $\tilde{p}(W)$ is an open neighborhood of $\tilde{x}$. Thus, so is $U:=\tilde{p}(W) \cap \tilde{r}^{-1}(V) \subset X / \widetilde{R}$. We can show that $U$ satisfies the above statement. Let $\tilde{y}, \tilde{z}$ be any distinct points in $U$. Then there exist two distinct points $y, z$ in $X$ such that $\tilde{p}(y)=\tilde{y}, \tilde{p}(z)=\tilde{z}$. If $\psi(y) \neq \psi(z)$, we have $f \in \Gamma\left(0, y_{0} 0\right)$ with $f \circ \psi(y) \neq f \circ \psi(z)$. And $f \circ \psi \in \Gamma\left(W, x_{0} 0\right)$ is constant on $A$. Put 


$$
F(\tilde{w}):= \begin{cases}f \circ \psi \circ(\tilde{p} \mid W-A)^{-1}(\tilde{w}), & \text { for } \tilde{w} \in \tilde{p}(W-A), \\ f\left(y_{A}\right), & \mid \text { for } \tilde{w} \in \tilde{p}(A) .\end{cases}
$$

Then $F \in \Gamma\left(\tilde{p}(W),{ }_{X} O / \tilde{R}\right) \subset \Gamma\left(U,{ }_{X} O / \widetilde{R}\right)$ and $f \circ \psi=F \circ \tilde{p}$ in $W$. Therefore $F(\tilde{y}) \neq F(\tilde{z})$. If $\psi(y)=\psi(z)$, then $y, z \in A$ and $p(y) \neq p(z)$. Hence we have $g \in \Gamma\left(V,{ }_{A} O / R\right)$ with $g \circ p(y) \neq g \circ p(z)$. Put in $U, G:=g \circ \tilde{r}$; then $G \in$ $\Gamma\left(U,{ }_{X} O / \widetilde{R}\right)$ with $G(\tilde{y}) \neq G(\widetilde{z})$, since $r: X \rightarrow A$ is a holomorphic retraction. Thus $\left(X / \widetilde{R},{ }_{X} O / \widetilde{R}\right)$ is locally morph-separable. Q.E.D.

REMARK 1 . We can easily find the examples such that $X / \widetilde{R}$ is not an analytic space, in the case that $R$ is not finite in (1), or $A$ is not contractible in (2), (3) respectively.

Corollary 1. Let $\left(X, X_{X} 0\right),\left(A,{ }_{A} 0\right)$ and $R$ be as in Theorem 1, (1) or (3). Then $A / R$ is embedded in $X / \widetilde{R}$. In particular, in the case of (3), $A / R$ is contractible and retractable in $X / \widetilde{R}$.

Proof. The canonical mapping $j: A / R \rightarrow j(A / R)$ is a holomorphic homeomorphism since $j$ is proper. We assert that for any $\tilde{a} \in A / R, j_{\tilde{a}}^{*}:\left({ }_{x} O / \widetilde{R}\right)_{j(\tilde{a})} \rightarrow$ $\left({ }_{A} \mathrm{O} / \mathrm{R}\right)_{\tilde{a}}$ is surjective.

(1) For any $f \in\left({ }_{A} O / R\right)_{\tilde{a}}(\tilde{a} \in A / R)$, we have $p_{a}^{*}(f) \in{ }_{A} 0_{a}\left(a \in p^{-1}(\tilde{a})\right)$. Then there exists $g \in_{X} O_{a}$ with $i_{a}^{*}(g)=p_{a}^{*}(f)$. Since $p$ is finite proper, we have $G \in\left({ }_{X} O / \widetilde{R}\right)_{j(\tilde{a})}$ with $\widetilde{p}_{a}^{*}(G)=g$. Then it follows that $j_{\tilde{a}}^{*}(G)=f$.

(3) Since $\tilde{r} \circ j=\mathrm{id}_{A / R}$, surjectiveness of $j_{\tilde{a}}^{*}$ is evident and in particular $\tilde{r}$ is a holomorphic retraction. Therefore $A / R$ is retractable and contractible in $X / \widetilde{R}$. Q.E.D.

3. Applications. We now consider the following problem: Let $\left(X,{ }_{X} 0\right)$ and $\left(M,{ }_{M}\right)$ be analytic spaces, $A$ a nowhere dense analytic set in $X$ and $h$ : $A \rightarrow M$ a surjective proper holomorphic mapping. Then, does an analytic space $Y$ exist with the following property (P)?

(P) There exist a surjective proper holomorphic mapping $\tilde{h}: X \rightarrow Y$ and an injection $j: M \rightarrow Y$ such that the restriction $\tilde{h} \mid A=j \circ h$ and $\tilde{h} \mid(X-A) \rightarrow$ $(Y-\tilde{A})(\tilde{A}:=\tilde{h}(A))$ is biholomorphic.

DEFinition 7. We say that a reduced analytic space $X$ is maximal if, for any open set $U \subset X$ and a nowhere dense analytic set $S \subset U$, every continuous function on $U$ which is holomorphic on $U-S$ is actually holomorphic on $U$.

REMARK 2. If an analytic space $\left(X,{ }_{X} 0\right)$ is maximal, $x_{X} 0$ is the maximal reduced complex structure on $X$. is $X / \widetilde{R}$.

Let $X, A$ and $R$ be as in Theorem 1 (1) or (2) or (3). If $X$ is maximal, so 
Let $R_{h}$ be the equivalence relation on $A$ defined by $h: A \rightarrow M$ (i.e. for any $u, v \in A, u R_{h} v$ means $h(u)=h(v)$ ). Then $R_{h}$ is proper and, if $M$ is maximal we can show that $A / R_{h}, M$ are isomorphic. Thus from Theorem 1 and Corollary 1 , we have

THEOREM 2. If (1) or (3) in Theorem 1 is satisfied for $X, A, R_{h}$ and $M$ is maximal, there exists an analytic space $Y$ with the property $(\mathrm{P})$.

Corollary 2. Let $X, A, M$ and $R_{h}$ be as in Theorem 2. Suppose that $X$ is maximal. Then any maximal analytic space $Y^{\prime}$ with the property $(\mathrm{P})$ is biholomorphically equivalent to $X / \widetilde{R}_{h}$.

Proof. Let $\tilde{p}^{\prime}: X \rightarrow Y^{\prime}$ be a surjective proper holomorphic mapping and $j^{\prime}: A / R_{h} \rightarrow Y^{\prime}$ an injection such that the restriction $\tilde{p}^{\prime} \mid A=j^{\prime} \circ p$ and $\tilde{p}^{\prime} \mid(X-A)$ $\rightarrow\left(Y^{\prime}-\tilde{p}^{\prime}(A)\right)$ is biholomorphic. Then, from Lemma 1 , we have the unique holomorphic mapping $\psi: X / \widetilde{R}_{h} \rightarrow Y^{\prime}$ with $\tilde{p}^{\prime}=\psi \circ \tilde{p}, j^{\prime}=\psi \circ j$.

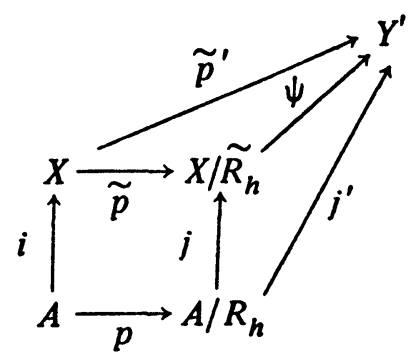

Since the restriction $\psi \mid\left(X / \tilde{R}_{h}-\tilde{p}(A)\right) \rightarrow\left(Y^{\prime}-\tilde{p}^{\prime}(A)\right)$ and $\psi \mid j\left(A / R_{h}\right) \rightarrow$ $j^{\prime}\left(A / R_{h}\right)$ are biholomorphic, $\psi$ is bijective. Moreover, $\psi^{-1}$ is continuous since $\widetilde{p}^{\prime}$ is proper. Hence $\psi$ is a holomorphic homeomorphism. By assumption, $Y^{\prime}$ is maximal, thus $\psi$ is biholomorphic. Q.E.D.

Now, using Theorem 1, (3), we shall extend Satz 1 of H. Kerner [8]. Let $X_{k}$ be a connected complex manifold and $A_{k}$ a contractible and retractable analytic set in $X_{k}$. Let $R_{k}$ be an equivalence relation on $A_{k}$ such that $A_{k} / R_{k}$ is an analytic space and $\operatorname{dim}_{a} R_{k}(a)>0$ for any $a \in A_{k}(k=1,2)$. If $R_{k}$ is proper, $X_{k} / \widetilde{R}_{k}$ is an analytic space and the natural projection $\tilde{p}_{k}: X_{k} \rightarrow X_{k} / \tilde{R}_{k}$ is proper holomorphic. Let $r_{k}: X_{k} \rightarrow A_{k}$ be the holomorphic retraction. Then we use the following result.

Lemma 2 (H. HolmanN [5]). Let $X$ be a complex manifold and $A$ an analytic set in $X$. Suppose that $r: X \rightarrow A$ is a holomorphic retraction. Then $A$ is a closed complex submanifold of $X$ and, for any $a \in A$, there exists an open neighborhood $U \subset X$ such that the restriction $r \mid U$ is a holomorphic projection (i.e. there exist two complex manifolds $M_{1}, M_{2}$ and a biholomorphic mapping $T: U \rightarrow M_{1} \times M_{2}$ such that $\mathrm{pr}=T \circ r \circ T^{-1}$, where $\mathrm{pr}: M_{1} \times M_{2} \rightarrow M_{1} \times$ 
$M_{2}, \operatorname{pr}\left(x_{1}, x_{2}\right)=\left(x_{1}, x_{2}^{0}\right)$ for any $\left(x_{1}, x_{2}\right) \in M_{1} \times M_{2}, x_{2}^{0}$ is a fixed point $)$.

If $\varphi$ is a holomorphic mapping of an analytic space $X$ into an analytic space $Y$, we put f.m.d. $\varphi:=\min _{x \in X} \operatorname{dim}_{x} \varphi^{-1}(\varphi(x))$. Then using Lemma 2 and the assumption $\operatorname{dim}_{a} R_{k}(a)>0$, we can prove the next lemma in almost like manner as in [8].

Llimma 3. Suppose that f.m.d. $r_{k} \geqslant 2$. Then $\tilde{A}_{k}:=\widetilde{p}_{k}\left(A_{k}\right)$ is the set of all singular points of $X_{k} / \widetilde{R}_{k}$.

Thiorem 3. Suppose that f.m.d. $r_{2} \geqslant \operatorname{dim} A_{1}+2$. If $X_{1} / \tilde{R}_{1}$ and $X_{2} / \tilde{R}_{2}$ are analytically equivalent, the following diagrams $(k=1,2)$ are analytically equivalent.

Proor. We first show that

$$
\text { f.m.d. } r_{1} \geqslant \operatorname{dim} A_{2}+2
$$

in some open neighborhood of $A_{1}$.

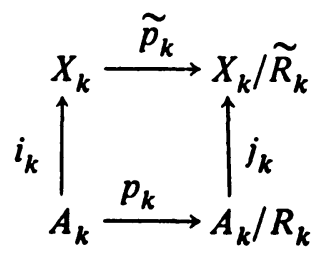

By assumption, any point of $A_{k}(k=1,2)$ has an open neighborhood with the property stated in Lemma 2. Let $O_{k}$ be the union of all such open neighborhoods. Then

$$
\operatorname{dim} O_{2}-\operatorname{dim} A_{2} \geqslant \text { f.m.d. } r_{2} \geqslant \operatorname{dim} A_{1}+2 .
$$

Since $\operatorname{dim} O_{1}=\operatorname{dim} O_{2}$, it follows that

$$
\text { f.m.d. }\left(r_{1} \mid O_{1}\right)=\operatorname{dim} O_{1}-\operatorname{dim} A_{1} \geqslant \operatorname{dim} A_{2}+2 \text {. }
$$

Hence, by Lemma $3, \tilde{A}_{k}:=\tilde{p}_{k}\left(A_{k}\right)(k=1,2)$ is the set of all singular points of $X_{k} / \widetilde{R}_{k}$. Let $\psi: X_{1} / \widetilde{R}_{1} \rightarrow X_{2} / \widetilde{R}_{2}$ be the biholomorphic mapping. Then $\psi\left(\tilde{A}_{1}\right)=\tilde{A}_{2}$ and there exists an open neighborhood $U_{k} \subset X_{k} / \tilde{R}_{k}$ of $\tilde{A}_{k}$ with $U_{k}^{\hat{k}}:=\tilde{p}_{k}^{-1}\left(U_{k}\right) \subset O_{k}$.

We now assert that there exists a holomorphic mapping $\psi^{\wedge}: U_{1}^{\wedge} \rightarrow U_{\mathbf{2}}^{\wedge}$ such that $\psi \circ \tilde{p}_{1}=\tilde{p}_{2} \circ \psi^{\curvearrowright}$. We put

$$
\begin{aligned}
& \psi^{\nu}:=\psi \mid\left(U_{1}-\tilde{A}_{1}\right) \rightarrow\left(U_{2}-\tilde{A}_{2}\right), \\
& \tilde{p}_{k}^{\sim}:=\tilde{p}_{k} \mid\left(U_{k}^{\hat{-}}-A_{k}\right) \rightarrow\left(U_{k}-\tilde{A}_{k}\right) \quad(k=1,2) .
\end{aligned}
$$


These mappings are biholomorphic. And we put, on $U_{1}^{\hat{1}}-A_{1}, \tau:=r_{2}$ 。 $\left(\tilde{p}_{2}^{\varsigma}\right)^{-1} \circ \psi^{\sim} \circ \tilde{p}_{1}^{\curlyvee}$. Then $\tau:\left(U_{1}^{\wedge}-A_{1}\right) \rightarrow A_{2}$ is also holomorphic. Since f.m.d. $\tau \geqslant \operatorname{dim} A_{1}+2$ on $U_{1}^{\wedge}-A_{1}$, we have the holomorphic mapping $\tilde{\tau}: U_{1}^{\hat{n}}$ $\rightarrow A_{2}$ such that $\tilde{\tau} \mid\left(U_{1}^{\wedge}-A_{1}\right)=\tau\left[9\right.$, Satz 2]. Define the mapping $\psi^{\wedge}: U_{1}^{\wedge}$ $\rightarrow U_{2}^{\hat{n}}$ as follows:

$$
\psi^{\wedge}(x)= \begin{cases}\left(\tilde{p}_{2}^{\sim}\right)^{-1} \circ \psi^{\sim} \circ \tilde{p}_{1}^{\sim}(x), & \text { for } x \in U_{1}^{\wedge}-A_{1}, \\ i_{2} \circ \tilde{\tau}(x), & \text { for } x \in A_{1},\end{cases}
$$

where $i_{2}: A_{2} \rightarrow U_{2}^{\wedge}$ is the injection. Remark that $\tilde{\tau}=r_{2} \circ \psi^{\wedge}$ on $U_{1}^{\wedge}$.

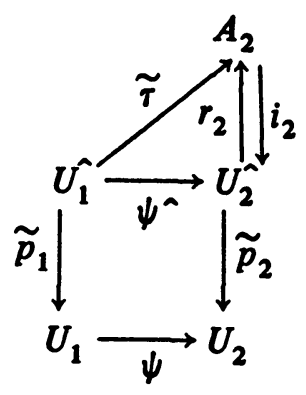

Then we can show that $\psi^{\wedge}: U_{1}^{\wedge} \rightarrow U_{2}^{\wedge}$ is continuous. To show this, it suffices to say that $\psi^{\wedge}$ is continuous at any $a \in A_{1}$, and hence, for any sequence $\left\{a_{n}\right\} \subset U_{1}^{\wedge}-A_{1}$ which converges to $a,\left\{\psi^{\wedge}\left(a_{n}\right)\right\}$ converges and $\lim _{n \rightarrow \infty} \psi^{\wedge}\left(a_{n}\right)$ $=\psi^{\wedge}(a)$.

$\left\{\psi^{\wedge}\left(a_{n}\right)\right\}=\left\{\tilde{p}_{2}^{-1}\left(\psi \circ \tilde{p}_{1}\left(a_{n}\right)\right)\right\} \subset U_{2}^{\wedge}-A_{2}$ has cluster points in $U_{2}^{\wedge}$ since $\tilde{p}_{2}$ is proper, and they must be contained in $A_{2}$. Further, the cluster points are unique and coincide with $\psi^{\wedge}(a)$. In fact, if $\alpha$ is a cluster point of $\left\{\psi^{\wedge}\left(a_{n}\right)\right\}$, we have a subsequence $\left\{a_{n}^{\prime}\right\}$ of $\left\{a_{n}\right\}$ with $\lim _{n \rightarrow \infty} \tilde{p}_{2}^{-1} \circ \psi \circ \tilde{p}_{1}\left(a_{n}^{\prime}\right)=\alpha$. Then

$$
\begin{aligned}
\alpha & =r_{2}(\alpha)=r_{2}\left(\lim _{n \rightarrow \infty} \tilde{p}_{2}^{-1} \circ \psi \circ \tilde{p}_{1}\left(a_{n}^{\prime}\right)\right) \\
& =\lim _{n \rightarrow \infty} r_{2} \circ \tilde{p}_{2}^{-1} \circ \psi \circ \tilde{p}_{1}\left(a_{n}^{\prime}\right)=\lim _{n \rightarrow \infty} \tau\left(a_{n}^{\prime}\right) \\
& =\lim _{n \rightarrow \infty} \tilde{\tau}\left(a_{n}^{\prime}\right)=\tilde{\tau}(a)=\psi^{\wedge}(a) .
\end{aligned}
$$

Hence $\lim _{n \rightarrow \infty} \psi^{\wedge}\left(a_{n}\right)=\psi^{\wedge}(a)$. Therefore $\psi^{\wedge}$ is continuous. Since $U_{k}^{\wedge}$ is a complex manifold $(k=1,2)$ and $\psi^{\wedge} \mid\left(U_{1}^{\wedge}-A_{1}\right)$ is holomorphic on $U_{1}^{\wedge}-A_{1}$, $\psi^{\wedge}$ is holomorphic on $U_{1}^{\wedge}$. Further, $\psi \circ \widetilde{p}_{1}=\widetilde{p}_{2} \circ \psi^{\wedge}$ on $U_{1}^{\wedge}$.

To complete the proof of the theorem, it suffices to show that $\psi^{\wedge}$ is bijective and its inverse is holomorphic. By (*), we also have the holomorphic mapping $\left(\psi^{-1}\right)^{\wedge}: U_{2}^{\wedge} \rightarrow U_{1}^{\wedge}$ such that $\psi^{-1} \circ \widetilde{p}_{2}=\widetilde{p}_{1} \circ\left(\psi^{-1}\right)^{\wedge}$ on $U_{2}^{\wedge}$. Then it follows that 


$$
\begin{aligned}
& \left(\psi^{-1}\right)^{\wedge} \circ \psi^{\wedge}=\text { id } \text { on } U_{1}^{\wedge}, \\
& \psi^{\wedge} \circ\left(\psi^{-1}\right)^{\wedge}=\text { id } \text { on } U_{2}^{\wedge} .
\end{aligned}
$$

Hence $\psi^{\wedge}: U_{1}^{\wedge} \rightarrow U_{2}^{\wedge}$ is biholomorphic and, in particular, $\psi^{\wedge}\left(A_{1}\right)=A_{2}$. Therefore $A_{k}, X_{k}$ and $A_{k} / R_{k}(k=1,2)$ are analytically equivalent respectively, and the two diagrams are analytically equivalent. Q.E.D.

REMARK 3. H. Kerner [8] has treated the case that $r_{k}: X_{k} \rightarrow A_{k}$ $(k=1,2)$ is a weakly negative vector bundle and $R_{k}(a)=A_{k}$ for any $a \in A_{k}$.

\section{REFERENCES}

1. H. Cartan, Quotients of complex analytic spaces, Contributions to Function Theory, (Internat. Collog. Function Theory, Bombay, 1960), Tata Inst. of Fundamental Research, Bombay, 1960, pp. 1-15. MR 25 \#3199.

2. H. Grauert, Uber Modifikationen und exzeptionelle analytische Mengen, Math. Ann. 146 (1962), 331-368. MR 25 \#583.

3. H. Grauert and R. Remmert, Konvexität in der komplexen Analysis. Nicht-holomorph. konvexe Holomorphiegebiete und Anwendungen auf die Abbildungstheorie, Comment. Math. Helv. 31 (1956), 152-183. MR 19, 456.

4. H. Holmann, Komplexe Räume mit komplexen Transformationsgruppen, Math. Ann. 150 (1963), 327-360. MR 27 \#776.

5. - Zur Regularität holomorpher Abbildungen zwischen komplexen Räumen, Math. Ann. 172 (1967), 17-32. MR 36 \#429.

6. B. Kaup, Uber Kokerne und Pushouts in der Kategorie der komplex-analytischen Räume, Math. Ann. 189 (1970), 60-76. MR 42 \#7944.

48-64. MR 44 \#511.

7. - Relationen auf komplexen Räumen, Comment. Math. Helv. 46 (1971), \#11566.

8. H. Kerner, Deformierbare Singularitäten, Math. Ann. 199 (1972), 337-344. MR 48

9. K. Stein, Fortsetzung holomorpher Korrespondenzen, Invent. Math. 6 (1968), 7890. MR 38 \#1286.

10. K. Takijima, Direct limits and inverse limits of equivalence relations on analytic spaces, Sci. Rep. Tokyo Kyoiku Daigaku 11 (1972), 143-148. MR 49 \#5404.

11. - On the continuation of equivalence relations on analytic spaces, $\mathrm{J}$. Saitama Univ. (to appear).

12. K.-W. Wiegmann, Strukturen auf Quotienten komplexer Räume, Comment. Math. Helv. 44 (1969), 93-116. MR 39 \#3032.

DEPARTMENT OF MATHEMATICS, FACULTY OF EDUCATION, SAITAMA UNIVERSITY, URAWA, JAPAN 